\title{
Study of Method of Proper Placement of DJ Stent during Open Pyeloplasty in Children for Uretero Pelvic Junction Obstruction, Without C-Arm
}

\author{
Author \\ Dr Nirmal Bhaskar \\ MS, MCh Associate Professor, Department of Paediatric Surgery \\ Govt Medical College, Thrissur, Kerala \\ Corresponding Author \\ Dr Nirmal Bhaskar \\ Nirmallya House, Gayathri Avenue, Koorkenchery .P.O., Thrissur, 680007 Kerala INDIA \\ Email: nirmalbhaskar321@gmail.com,Phone 9846898030
}

\begin{abstract}
Introduction: Uretero Pelvic junction (UPJ) obstruction is defined as an obstruction of the flow of urine from the renal pelvis to the proximal ureter. Pyeloplasty is the treatment. Proper placement of DJ stent is usually confirmed with C-Arm.

Objective: Study of Method of Proper Placement of DJ Stent During Open Pyeloplasty in Children for UreteroPelvic Junction Obstruction, Without C-Arm.

Materials and Methods: This is a prospective study of 3 years duration. Total there were 20 children. Only Anderson-Hynes dismembered pyeloplasty included in the study. C-Arm is not used. Our method involved opening renal pelvis and UPJ obstruction is resected. 6FG infant feeding tube is introduced to ureter and $15 \mathrm{cc}$ Normal saline injected. Then DJ stent is put via opening at upper end of ureter. Now apply pressure on bladder. If saline is coming through the upper end we can confirm the position of DJ stent.

Result: Of the total 20 cases, we put DJ stent in 17 cases. In 3 cases there was difficulty in introducing DJ stent and saline didn't come back. All the 17 cases had successful removal of DJ stent with cystoscope.

Conclusion: Our study shows that the above method is a good option for confirming the position of DJ stent, without $C$ - Arm and radiation. All DJ stents can be removed by cystoscopy. It can avoid unnecessary exploration of kidney and ureter to remove stent.

Keywords: Open Pyeloplasty, Uretero Pelvic Junction Obstruction, DJ stent, C-Arm, Cystoscopy, Anderson Hynes Pyeloplasty.
\end{abstract}

\section{Introduction}

Ureteropelvic junction (UPJ) obstruction is defined as an obstruction of the flow of urine from the renal pelvis to the proximal ureter.

UPJ obstruction is the most common cause of neonatal and antenatal hydronephrosis, occurring in one per 1500 live births. Prior to the use of prenatal ultrasonography, most patients with UPJ obstruction presented with pain, hematuria, urosepsis, failure to thrive, or a palpable mass.

UPJ obstruction is associated with a number of anomalies. A known association exists between UPJ obstruction and horseshoe kidneys. ${ }^{[1]}$ UPJ obstruction has also been found to occur in 17 of 82 
patients with ectopic kidneys. A strong relationship also exists between UPJ obstruction and nephrolithiasis. ${ }^{[2]}$ One study found a $20 \%$ incidence rate of stones in patients with UPJ obstruction. Finally, no strong evidence exists indicating a hereditary pattern. Only one study has suggested that UPJ obstruction has a genetic etiology, ${ }^{[3]}$ and thus, most cases are believed to be spontaneous in nature.

Clinical symptoms of UPJ obstruction later in life include urosepsis, failure to thrive, flank pain or mass, and hematuria. As first described by Dietl, the episodes of flank pain, nausea, and vomiting may manifest during periods of rapid diuresis with large volumes of liquid intake (so-called Dietl crisis). ${ }^{[4]}$ Pyeloplasty is the surgical reconstruction or revision of the renal pelvis to drain and decompress the kidney. Most commonly it is performed to treat an uretero-pelvic junction obstruction if residual renal function is adequate.

While open pyeloplasty is still considered the criterion standard for treatment of UPJ obstruction in infants, laparoscopic pyeloplasty, with or without robotic assistance, is the treatment of choice in older children and in most adults. ${ }^{[5]}$

In the pediatric population, although only a few published series have addressed long-term outcomes, the short-term data suggest similar success in both traditional open and laparoscopic pyeloplasty, including treatment of the youngest children. ${ }^{[6,7]}$

Open pyeloplasty remains the criterion standard for the treatment of UPJ obstruction. Although many variations exist in the methodology, this procedure typically involves the surgical excision of the narrowed segment of the UPJ and performance of a spatulated reanastomosis of the renal pelvis to the ureter. If significant dilation of the renal pelvis occurs, it is often reduced in size by trimming off redundant tissue, and then it is tailored in such a fashion that it funnels down towards the anastomosis. If an accessory or aberrant vessel exists near the UPJ, the anastomosis is positioned anterior to the vessel.

An open pyeloplasty can be performed through a variety of incisions but is most likely performed through an extraperitoneal flank incision.
Depending on the surgeon's preference and the function of the kidney postoperatively, a nephrostomy tube is occasionally left in place, or, more often, a ureteral DJ stent that passes from the renal pelvis to the bladder is placed

Usually complete length of DJ stent is introduced through the upper end of ureter. Lower end of DJ stent will reach bladder, and upper end of ureter is put in the renal pelvis and anastomosis is completed. The proper position of DJ stent will be inside the ureter in such a way that, the upper end will be in renal pelvis, and lower end will be in bladder. DJ stent will be occupying a transanastomotic position. Usually C-Arm is used to confirm the position. CArm will confirm whether the lower end of DJ stent reached bladder. Because DJ stent is removed later by doing cystoscopy. If the lower end is not reached at bladder, then removal of DJ stent will be difficult. Method of putting the DJ stent is first fill the bladder with saline, by introducing infant feeding tube via upper end of ureter to bladder and injecting saline to bladder. Then introduce complete length of DJ stent through upper end of bladder. Then apply pressure over bladder. If saline come out through the upper end of DJ stent from bladder, we can confirm that the lower end reached the bladder. Once the position of DJ stent is confirmed, we can complete the anastomosis

Some surgeons leave a drain near the anastomosis; this is removed postoperatively when the output becomes minimal. The rationale behind this is to help detect fluid leakage from the anastomosis and to prevent its accumulation in the retroperitoneum. If a drain is left, it is usually removed after the patient's bladder Foley catheter is removed and after trial of having the nephrostomy tube (if present) clamped to ascertain that neither maneuver will increase drainage from the anastomosis.

The advantages of this operation include excellent exposure of the UPJ, familiar anatomy for essentially all urologists, the ability to tailor the renal pelvis as needed, and the performance of a watertight anastomosis. The disadvantages include the large surgical incisions and the associated postoperative pain and convalescence. 


\section{Objective}

Study of Method of Proper Placement of DJ Stent During Open Pyeloplasty in Children for Uretero Pelvic Junction Obstruction, Without C-Arm.

\section{Materials and Methods}

This is a prospective study of 3 years duration. Total there were 20 children. Only Anderson-Hynes dismembered pyeloplasty included in the study.CArm is not used. After opening renal pelvis and UPJ obstruction is resected. $6 \mathrm{FG}$ infant feeding tube is introduced to upper end of ureter to the maximum length and $15 \mathrm{cc}$ Normal saline is injected to bladder. Then infant feeding tube is removed and DJ stent is put via opening at upper end of ureter. After complete insertion, the upper tip of DJ stent is put in renal pelvis. Now apply pressure on bladder. If saline is coming thorough the upper end we can confirm that the lower end is at bladder. Then complete the anastomosis.

\section{Results}

Of the total 20 cases, we put DJ stent in 17 cases. We did cystoscopy and removed DJ stents. In 3 cases there was difficulty in introducing DJ stent and saline didn't come back, while applying pressure over bladder. Those cases we put infant feeding tube as transanastomotic nephrostomy tube and upper end of the tube is kept transabdominally outside body. They can be removed from outside. DJ stents are removed after 2 months after surgery. All the 17 cases had successful removal of DJ stent.

\section{Discussion}

Our study shows that the above method is a good option for confirming the position of DJ stent, without C-Arm and radiation. All DJ stents which are put by this method can be removed by cystoscopy. It can avoid unnecessary exploration of kidney and ureter for DJ stent removal.

Once DJ stent is inserted and Saline not coming back on pressure means, sometimes DJ stent not reached bladder. Sometimes there may be additional Vesicoureteric Junction obstruction, which obstructs DJ stent. Those cases it is better to use C-ARM or do cystoscopy to find out the weather DJ stent reached bladder. Otherwise better to put infant feeding tube as transanastomotic nephrostomy tube and upper end of the tube is kept transabdominally outside body. They can be removed from outside.

\section{References}

1. Blanc T, Koulouris E, Botto N, Paye-Jaouen A, El-Ghoneimi A. Laparoscopic pyeloplasty on horseshoe kidney in children. J Urol. 2013 Oct 16. [Medline].

2. Naitoh Y, Kawauchi A, Kamoi K, Soh J, Hongo F, Okihara K, et al. Nephrolithotomy Performed Concurrently With Laparoendoscopic Single-site Pyeloplasty. Urology. 2013 Oct 19. [Medline].

3. Atwell JD. Familial pelviureteric junction hydronephrosis and its association with a duplex pelvicaliceal system and vesicoureteric reflux. A family study. Br J Urol. 1985 Aug. 57(4):365-9. [Medline]

4. Dietl J. Wandernde nieren und deren einklemmung. Wien Med Wochenschr. 1864. 14:153.

5. Symons SJ, Bhirud PS, Jain V, Shetty AS, Desai MR. Laparoscopic pyeloplasty: our new gold standard. J Endourol. 2009 Mar. 23(3):463-7. [Medline].

6. Casale P. Robotic pyeloplasty in the pediatric population. Curr Urol Rep. 2009 Jan. 10(1):55-9. [Medline]

7. Casale P. Robotic pyeloplasty in the pediatric population. Curr Opin Urol. 2009 Jan. 19(1):97-101. [Medline] 